\title{
An Experimental Investigation of the Transient Response of HTS Non-insulation Coil
}

\author{
Zhenyu Zhang1 • Chul Han Kim² • Jin Geun $\mathrm{Kim}^{2}$ • Jozef Kvitkovic ${ }^{2}$. \\ Sastry Pamidi ${ }^{2,3} \cdot$ Min Zhang ${ }^{1} \cdot$ Jianwei Li $^{1} \cdot$ Weijia Yuan ${ }^{1}$
}

Received: 10 April 2016 / Accepted: 24 September 2016 / Published online: 5 October 2016

(C) The Author(s) 2016. This article is published with open access at Springerlink.com

\begin{abstract}
A single pancake coil without turn-to-turn insulation was tested in this paper to investigate the transient responses under different situations. We performed charging and discharging test, $\mathrm{AC}$ current test, and regional quench emulation test on the non-insulated (NI) coil. The experimental test results show a significant time delay for charging and discharging characteristics of NI coil and can be validated by a simple proposed equivalent electrical circuit. Under the AC operating current, the NI coil can bypass nearly all the AC current from the coil spiral path to the radial path such that it is not possible for NI coil to store or be affected by the AC magnet field. Additionally, while carrying AC current, the AC loss dissipation of NI coil is inversely proportional to the frequency of the AC operating current. When a regional quench occurs, the NI coil can bypass the current in the regional quench zone to avoid further temperature accumulated and protect the NI coil itself.
\end{abstract}

\section{Zhenyu Zhang \\ zz279@bath.ac.uk \\ Weijia Yuan \\ w.yuan@bath.ac.uk}

1 Department of Electronic and Electrical Engineering, The University of Bath, Bath BA2 7AY, UK

2 Central for Advanced Power System, Florida State University, Tallahassee, FL 32310, USA

3 Department of Electrical and Computer Engineering, College of Engineering, Florida A\&M University-Florida State University College of Engineering (FAMU-FSU), Tallahassee, FL 32310, USA
Keywords AC current - Charging/discharging test . NI coil · Turn-to-turn characteristic resistance · Quench

\section{Introduction}

Firstly proposed in 2011, researchers have shown great interest to the non-insulation (NI) coil due to its superior performances over traditional insulated (INS) coils [1]. It is found that the self-protection of the NI coil has been improved based on the over-current test, which proves the enhanced thermal stability and simplifies the HTS magnet coil protection method [2]. In recent years, several papers have focused on the NI coil application study: the NI coil testing under time-varying background field was reported in [3], which indicates that the magnetic field of NI coil may not be proportional to the operating current. Also as reported in [4], it is suggested that the NI coil can be used as a low-pass filter. Charging, discharging, and overcurrent behaviors of the NI coil are investigated by using a comprehensive equivalent NI coil circuit grid model [5-8].

The protection of the HTS magnet coils is very crucial, it is necessary to continuously monitor the status of the HTS coils during the whole operation. In the case of quench occurring, the traditional method is to ensure the current diverted from the HTS coil to a dummy resistor. However, the NI coil has quite different electrical characteristics compared with its counterpart INS coil, which motivates the authors to investigate the transient response of the NI coil in order to develop an effective protection algorithm for HTS magnet coil. The outline of the paper is described as follows:

Firstly, the charging and discharging responses of NI coil differ significantly from the insulated coil. For large-scale magnet made from the NI coil, it may take hours to days in order to fully discharge the magnet. The time constant can 
characterize the total charging or discharging time of the NI coil and is mainly determined by the turn-to-turn mechanical contact tension. In this paper, the time constant of the proposed NI coil will be obtained from the experimental DC ramping current test and further verified by a simple electrical circuit.

Secondly, even if the superconducting magnet is charged with DC, there still exist AC losses generated from the AC ripple current offset by DC operating current. This part of dissipated heat should be carefully removed. There are several models to estimate the AC losses of the traditional INS coil [9], but the models to estimate the AC losses of the NI coil are not applicable since the NI coil provide an alternative radial path to bypass the AC current away from the original spiral path. Therefore, this paper will investigate the differences of AC losses between these two coil winding techniques in order to aid coil cooling design.

Thirdly, if a local quench occurs to the NI coil, in theory, the direct turn-to-turn contact can divert the current away from the local quench zone, which prevents further damage to the coil. Hence, in this paper, a local quench test can provide a novel method to prove that the thermal stability of NI coil is superior to the INS coil.

\section{Electrical Tests of NI and INS Coils}

A 30-turn single pancake coil was wound by reel-to-reel process using recently developed SuperPower advanced pinning (AP) HTS tape. For the comparison, an identical 30turn insulated single pancake coil as the counterpart was wound in the same method by using Kapton insulated HTS tape. Both coils were carefully wound at the same winding tension. Figure 1 shows the photo of the two coils. The critical currents of the two coils were experimentally measured

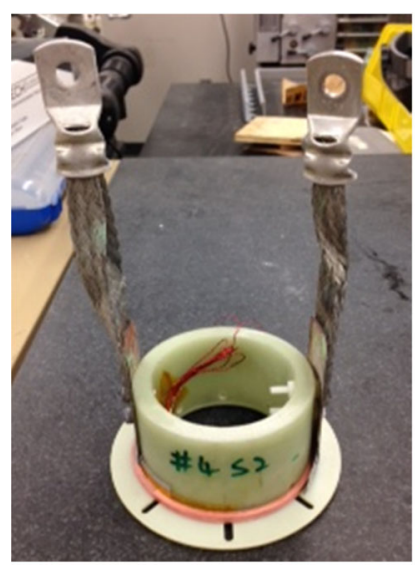

(a)

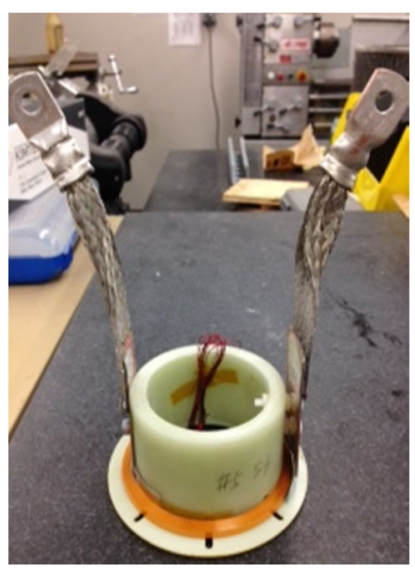

(b)
Fig. 1 The photo of the $\mathbf{a}$ NI and $\mathbf{b}$ INS coil

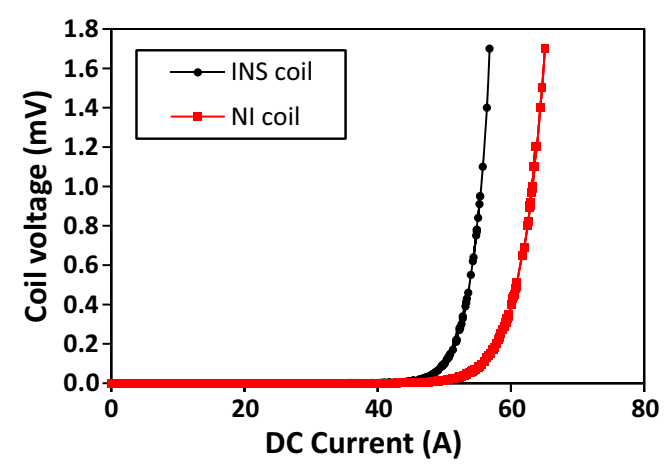

Fig. 2 I-V curves of the NI coil and INS coil

based on $1 \mu \mathrm{V} / \mathrm{cm}$ criterion by using voltage tap with 9-m interval; the results are shown in Fig. 2. Table 1 describes the detailed specifications of the both coils.

\subsection{Transient Response of the NI Coil Charged with DC Operating Current}

\subsubsection{Charging Test}

The NI coil was charged with DC ramping current with rates of 1,2 and $3 \mathrm{~A} / \mathrm{s}$ to $30 \mathrm{~A}$, respectively, and then the current was held at $30 \mathrm{~A}$ constantly. A hall sensor is placed at the center of the coil to measure the central magnet field of the NI coil. Figure 3 a block curves show the test results of the coil magnet field varies with the current. The magnet constant $k$ of the NI coil can be obtained: $k=0.40 \mathrm{mT} / \mathrm{A}$. Figure $3 \mathrm{~b}$ block curves show the test results of the coil terminal voltage varies with the current. A significant time

Table 1 The parameters of the NI and INS testing coils

\begin{tabular}{lll}
\hline Parameters & NI & INS \\
\hline Tape & & \\
HTS conductor & SuperPower; AP & \\
Overall width $(\mathrm{mm})$ & 4 & 4 \\
Thickness $(\mathrm{mm})$ & 0.1 & 0.22 \\
Ic of tape at $77 \mathrm{~K}$, self-field (A) & 98 & 112 \\
Coil & & \\
Ic of coil at $77 \mathrm{~K}$, self-field $(\mathrm{A})$ & 62.8 & 55.2 \\
$N$ value & 18.1 & 22 \\
Insulation & None & Kapton \\
Number of turns & 30 & 30 \\
I.D., O.D., $(\mathrm{mm})$ & $88.9,91.9$ & $88.9,95.5$ \\
Height $(\mathrm{mm})$ & 4 & 4 \\
Length $l(\mathrm{~m})$ & 8.8 & 9 \\
Inductance, $L_{\text {coil }}(\mu \mathrm{H})$ & 195 & 149 \\
\hline
\end{tabular}


Fig. 3 Charging test of NI coil at $30 \mathrm{~A}$ : a response of coil central magnet field and b response of coil terminal voltage response

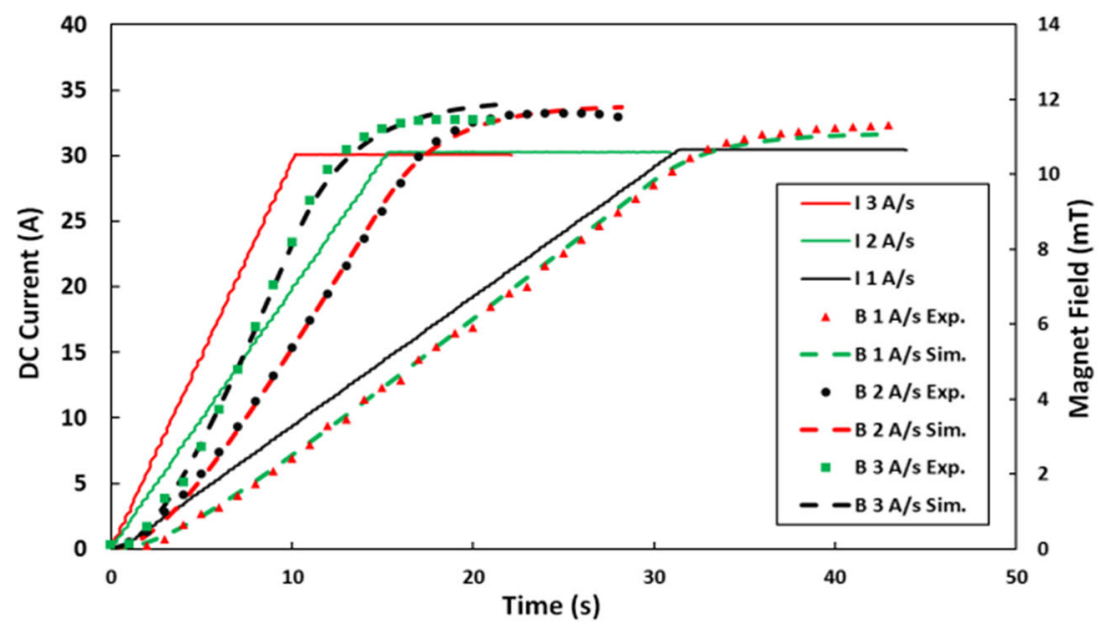

(a)

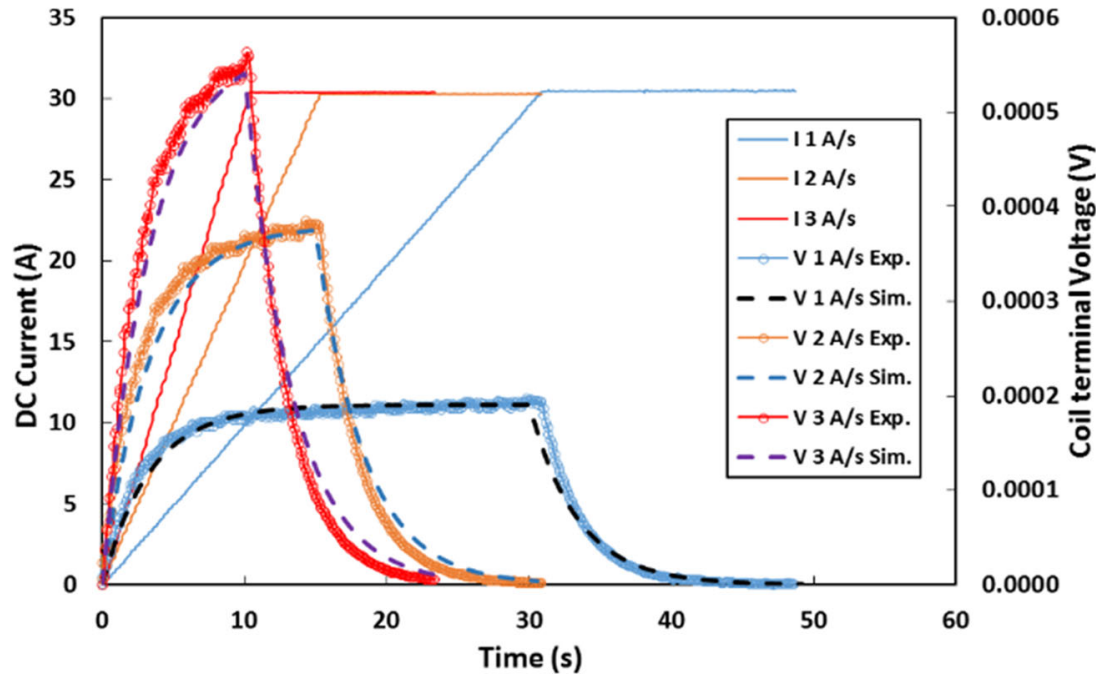

(b) delay of the magnet field and voltage responses are observed when the DC operating current is ramping up.

Unlike the INS coil, during the transient state, the NI coil can bypass the current from the original superconducting spiral path to the turn-to-turn radial path. Instead of pure superconductivity path, the current flowing through turnto-turn superconductivity layer will experience resistance contributed by copper stabilizer layer, Hastelloy substrate layer, and a buffer layer of the HTS tape. Hence, a characteristic resistance may be defined to describe the average turn-to-turn contact resistance $R_{c}$ of the NI coil.

A time constant $\tau$ can be utilized to describe the time delay of the magnet field and voltage of NI coils reaching the final stable value. Based on the measurement results in Fig. 3, Table 2 shows the values of the $\tau$. The characteristic resistance $R_{c}$ per meter of the NI coil can be calculated by $R_{c}=\frac{L_{\text {coil }}}{\tau \cdot l}$, where $l$ is the length of the HTS coil. The results are summarized in Table 2.
From the test results in Fig. 3b, it can be seen that the coil terminal voltage is gradually decreasing to zero when current is held steady at $30 \mathrm{~A}$. Assuming the zero resistance of the HTS tape, it means the current in radial direction is slowly flowing back to the spiral superconducting path resulting to a stable magnet field induced by the NI coil. Once the coil voltage reaches zero, it indicates the finishing of charging process. Hence, it can be seen that the charging time for the NI coil is inversely proportional to the charging rates. However, based on the results of Table 2, it can be seen that the time constant is independent of the charging

Table 2 The parameters of 30 turns NI coil

\begin{tabular}{llll}
\hline Parameters & $3 \mathrm{~A} / \mathrm{s}$ & $2 \mathrm{~A} / \mathrm{s}$ & $1 \mathrm{~A} / \mathrm{s}$ \\
\hline$\tau(\mathrm{ms})$ & 2930 & 3062 & 3064 \\
$R_{c}(\mu \Omega / \mathrm{m})$ & 7.4 & 7.1 & 7.1 \\
\hline
\end{tabular}


Fig. 4 Sudden discharging test of NI coil at 40-A steady-state operation

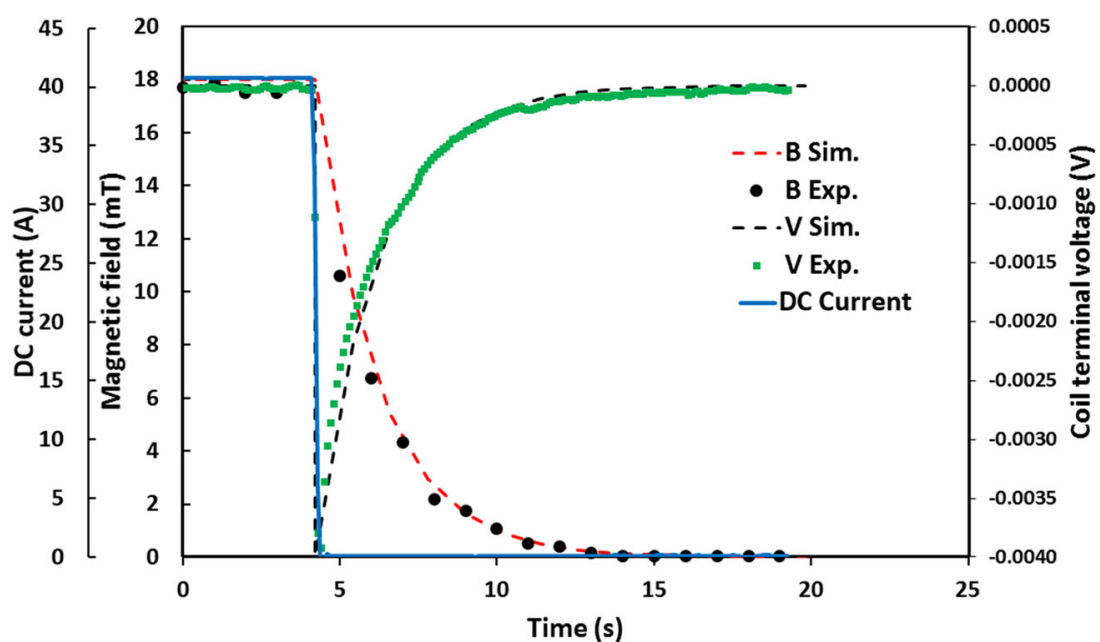

which consists of a characteristic resistance parallel with an inductor. The value of the inductor is obtained from experimental measurement, shown in Table 1, and the value of the characteristic resistance is calculated based on the time constant which represents the response of the NI coil reaching $63.2 \%$ to its final stable value. The simulation circuit is able to completely ignore the detailed configuration of the coil. The characteristic resistor represents the overall current path along the radial direction, and the inductor represents the overall current path along the spiral path. The largely simplified NI coil electrical circuit can quickly provide an estimated time for charging or discharging delay, which is particularly useful for designing a large-scale NI coil. The charging and sudden discharging of the simulation results are plotted in Figs. 3 and 4 as dashed curves, respectively. Good agreement was obtained between the experiment and simulation results, which validate the simplified NI coil electrical circuit.

\subsection{The Transient Response of NI Coil Charging with AC Operating Current}

Most DC power supply contains power converters, which often introduces ripple $\mathrm{AC}$ current at a frequency of several times higher than the power frequency. AC ripple current results in dissipating AC losses to the HTS coil [11]. In order to investigate the transient response of NI coil charged with AC operating current, the NI coil is connected with a frequency variable $\mathrm{AC}$ power source. For comparison, the INS coil as a counterpart is also charged with the same AC current. A hall sensor is placed at the center of the coil to measure the coil AC magnet field. In the case of NI coil charged with AC current, the heat dissipation resulted from the characteristic resistance of turn-to-turn contacts has made additionally major contribution to the total AC losses. In order to obtain the characteristic resistance under $\mathrm{AC}$ operating current, the AC losses of the NI and INS coils

Fig. 5 The simplified equivalent electrical representative circuit 


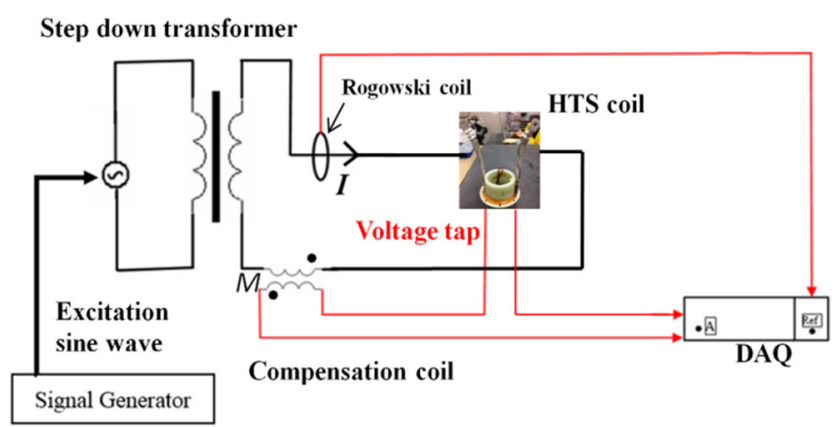

Fig. 6 Schematic of AC loss experimental test circuit

were measured at various frequencies based on the electrical method, the measurement circuit is shown in Fig. 6, and the measurement technique is based on previous work [12].

From the AC loss test results shown in Fig. 7, unlike the INS coil, the AC losses of the NI coil depend on the frequency and decrease with the increased frequency. This can be explained by the magnet field measurement results in Fig. 8. The magnet field of the INS coil can be measured by the hall sensor and it is frequency independent. On the other hand, the magnet field of the NI coil is hardly observed even in the low frequency that is less than $4 \mathrm{~Hz}$. For INS coil, the coil magnet field $B$ is mainly determined by the number of coil turns $N$ and the coil operating current $I$, i.e., $B$ is proportional to $\mu_{o} N I$. However, this is not the case for NI coil, there is no magnetic field stored in the NI coil. Hence, it can be concluded that nearly all the AC current in the NI coil is bypassed by the turn-to-turn radial path and the AC magnet field of the NI coil is significantly attenuated.

For the NI coil, higher frequency results in a lower AC loss value under the unit of joules/cycle, which is contrary to the hysteresis losses, the main component of AC losses [13]. Considering the magnitude of the AC losses being a function of the length of the INS coil, the current can only

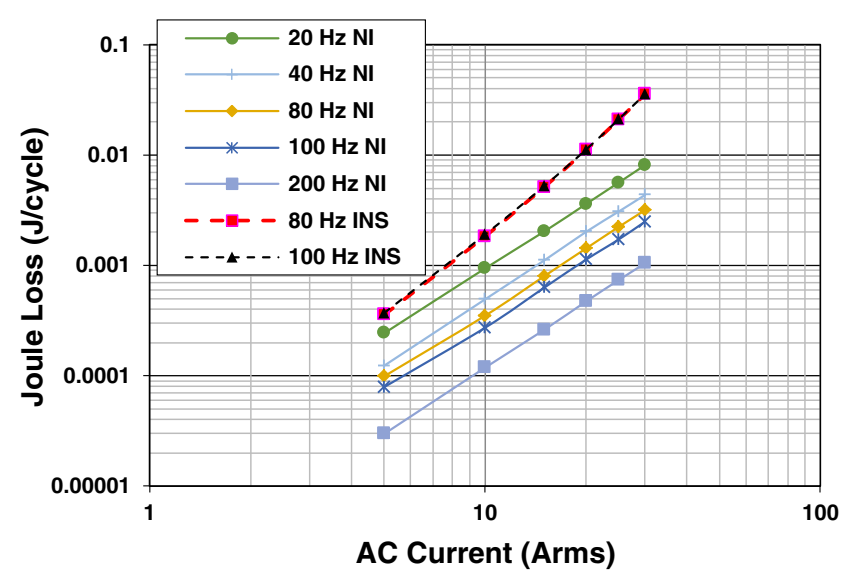

Fig. 7 The AC losses test results of the NI and INS coils at various frequencies

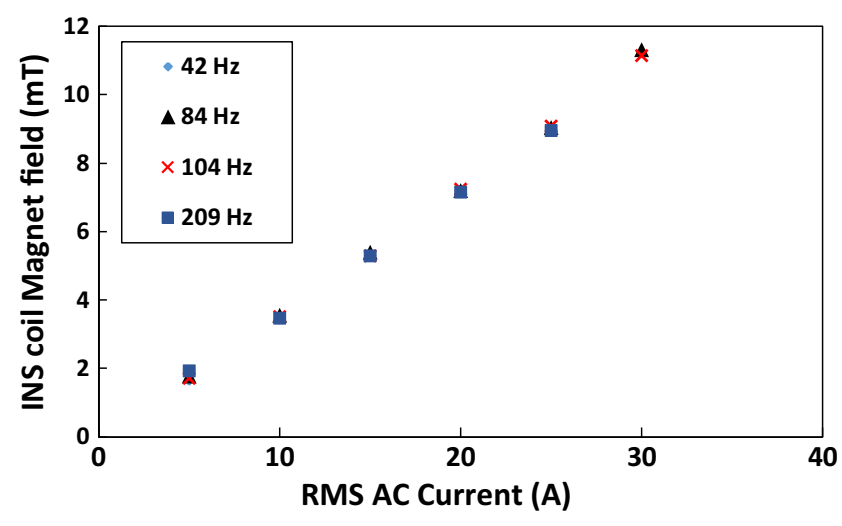

Fig. 8 The INS coil magnet field test results

flow along the whole length of the coil leading to the fixed coil length. Hence, the AC losses of INS coil are regardless of the frequency.

However, for the NI coil, the current can choose to flow along the coil spiral path or radial path depending on the impedance. Naturally, the current will choose the path with lower impedance. The impedance of the NI coil has two possible sources: the resistance due to turn-to-turn contact and the impedance due to coil inductance. Higher frequency will increase the impedance due to coil inductance and the coil winding tension will affect the turn-to-turn contact resistance. Additionally, the NI fabrication process is hard to ensure that the winding tension can keep uniform at every point; therefore, it can expect that at a higher frequency, the current will flow through both spiral path and radial path depending whichever the impedance is lower. Overall, as the results in Fig. 9, the turn-to-turn characteristic resistance of NI coil decreases with the increased frequency of AC current, which indicates that the AC current tends to flow along the shortest path to avoid the high impedance path in the NI coil.

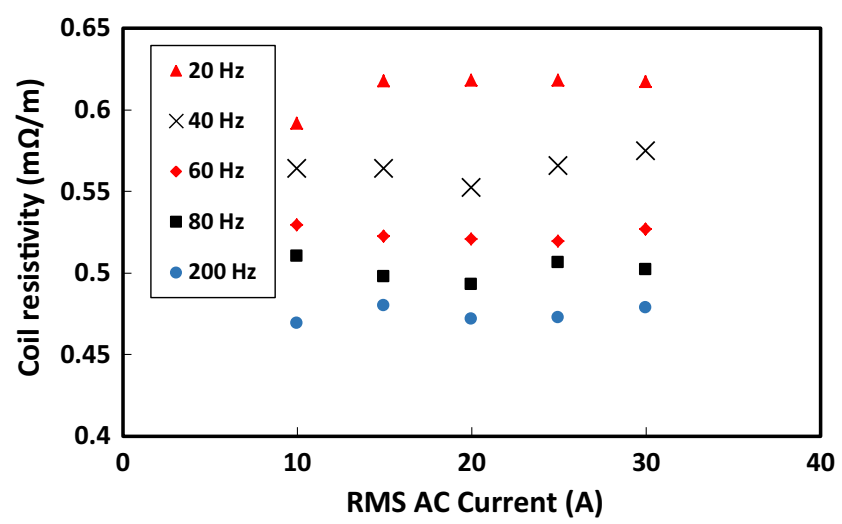

Fig. 9 The turn-to-turn characteristic resistance of the NI coil charged with AC operating current 


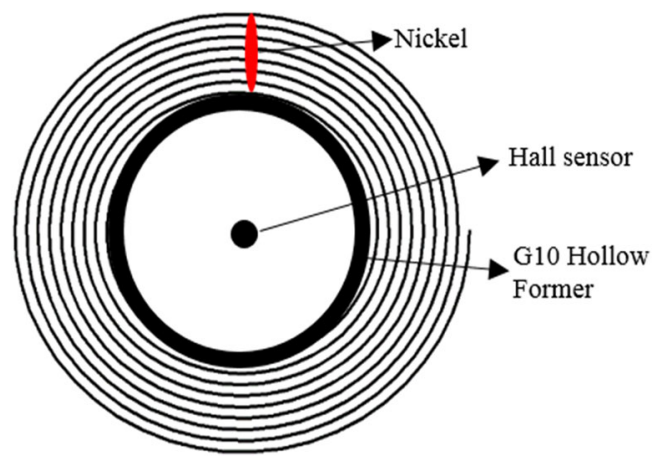

Fig. 10 The top view of coil with a heater under a small region of the coil

\subsection{The Transient Response of NI Coil with Regional Hotspot Under DC Operating Current}

The NI coil has been proved to be able to enhance the thermal stability when the current exceeds 1.2 times of the critical current compared with the INS coil [1]. However, when a quench occurs in a small part of the HTS coil, the heat generated from this hotspot is difficult to spread out due

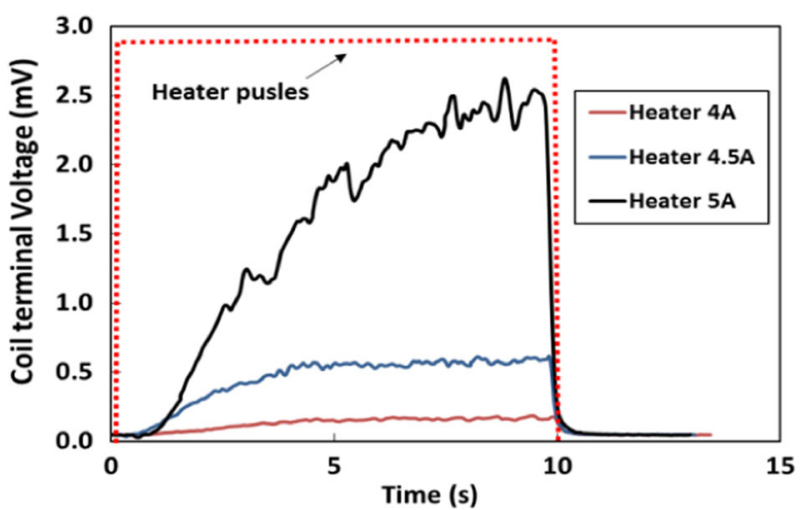

(a)

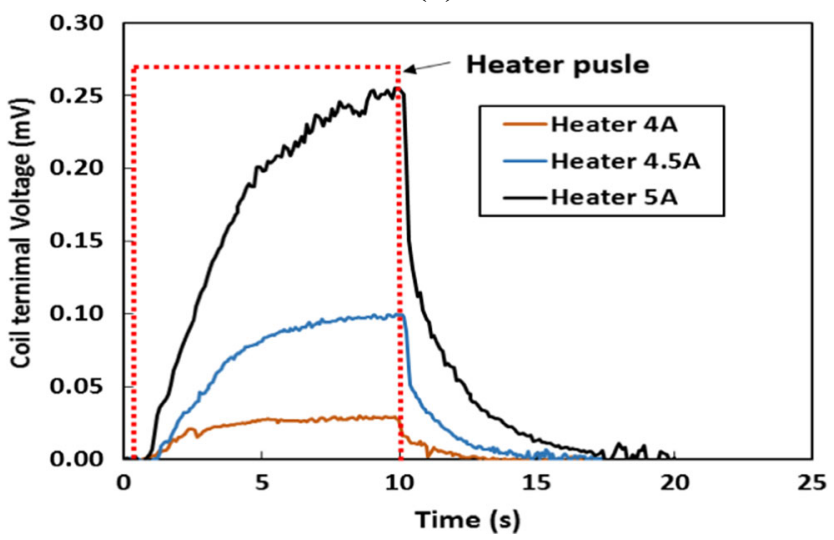

(b)

Fig. 11 The terminal voltage of the INS coil (a) and NI coil (b) with quench initiated by 4-, 4.5- and 5 A heater pulses, respectively, with coils are stable at 50-A DC operating current to the low thermal conductivity of HTS compared with LTS. The heat generated from the quench can only dissipate in a small regional area and results to the HTS tape burning out if the temperature exceeds $480 \mathrm{~K}$ [14]. However, the NI coil is able to provide the alternative radial path for the current to bypass the quench region, where the superconducting layer becomes highly resistive. To compare the quench behavior of the NI coil and the INS coil, nickel-chromium alloy wire is used as heater placed under the coil as shown in Fig. 10 to emulate a regional quench occurring in the coil. A hall sensor is placed at the center of the coil, and coil terminal voltage is measured by taking advantage of its fast response speed under external turbulence to the coil.

When the coil was charged with 50-A DC current and became steady-state for a sufficient time, a 10-s heating pulse is generated from a nickel wire to initiate a quench in the NI and INS coil, respectively. A nickel is powered by a DC power supply with currents of $4 \mathrm{~A}(48 \mathrm{~J}), 4.5 \mathrm{~A}$ $(60.75 \mathrm{~J})$, and $5 \mathrm{~A}(75 \mathrm{~J})$. Figure 11a for INS coil and $\mathrm{b}$ for NI coil shows the coil terminal voltage after the quench initiated by the nickel.

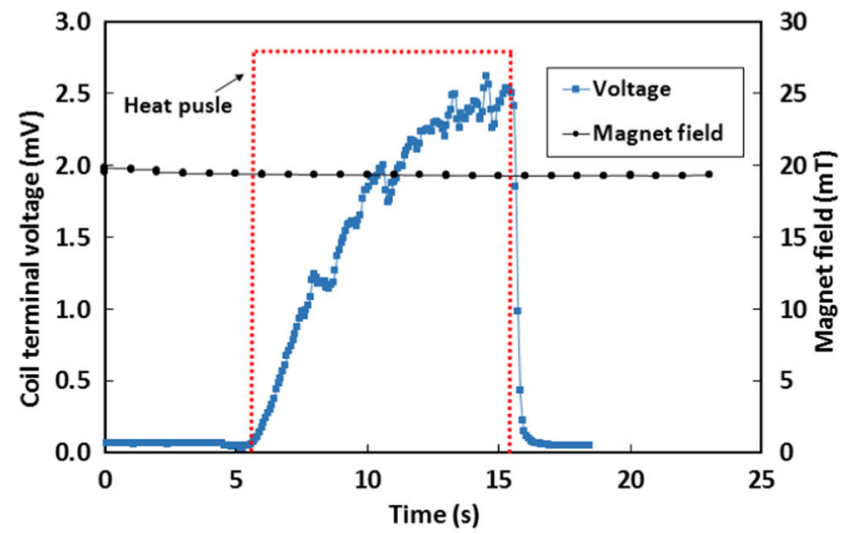

(a)

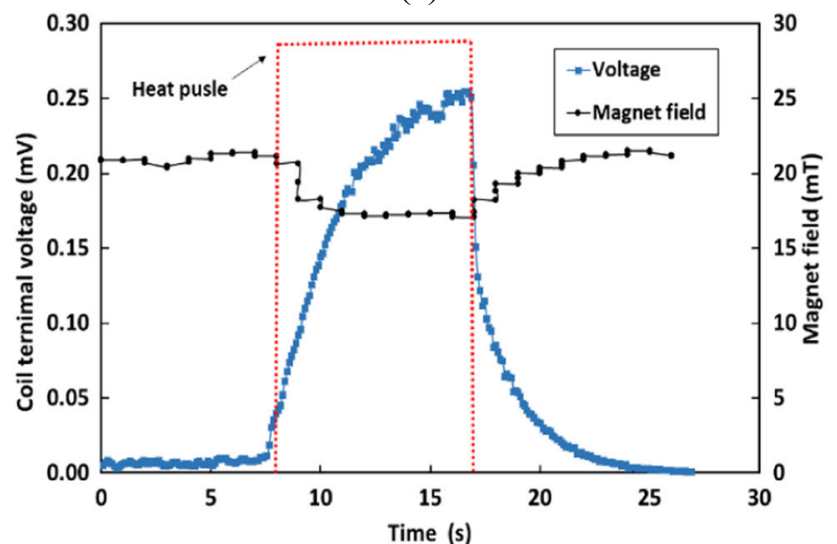

(b)

Fig. 12 The coil magnet field and terminal voltage test results of the INS coil (a) and NI coil (b) with quench initiated by 5-A heater pulse, with coils are stable at 50-A DC operating current 
As the results shown in Fig. 11, there are two main experimental results which are able to clarify the phenomena that the NI coil radial path can bypass the current if local quench occurs. The voltage of the insulated coil rises about 10 times higher than the non-insulated coil at the same level of the heat generated by the heater. This is because the current keeps flowing in the spiral path, which accelerates the quench propagation resulting in voltage increasing rapidly. However, the NI coil radial path can bypass the current away from the local quench zone resulting in voltage increasing slowly.

Additionally as in Fig. 12, hall sensors are placed in the central of the both NI coil and INS coil, respectively. Since the magnetic field of the coil is proportional to the coil spiral current and the number of turns, the magnetic field will decrease only when the coil spiral current decreases assuming that the number of turns is fixed. During the regional quench, the factor that the magnetic field of the NI coil decreases about $18 \%$ while the magnetic field of the insulated coil is unchanged indicates the NI coil radial path is able to bypass the current to avoid the regional quench zone.

\section{Conclusions}

This paper presents an experimental investigation on the dynamic responses on the NI coil under the different electrical transient states: charging and sudden discharging tests, $\mathrm{AC}$ current tests, and quench test. For the comparison, the counterpart of the NI coil, the INS coil has also been performed with the similar tests, and the testing conclusions may be summarized as follows:

1. The NI coil can be charged or discharged in order to store or release electromagnetic energy with DC current as normal INS coils under the steady-state. However, due to the turn-to-turn radial current diverted path, a significant time delay of the coil terminal voltage and magnet field responses will affect the transient characteristic of the NI coil.

2. The NI coil cannot store electromagnetic energy under the $\mathrm{AC}$ current. As nearly all the $\mathrm{AC}$ current will be diverted from the spiral path to the radial turn-to-turn path to avoid the large coil inductive impedance Hence, the AC magnet field is largely attenuated

3. During the coil regional quench occurring, the NI coil is able to divert the coil current to the radial path to avoid the highly resistive quench zone, which proves the thermal stability is enhanced by the NI coil under the thermal vibration cases.
Open Access This article is distributed under the terms of the Creative Commons Attribution 4.0 International License (http:// creativecommons.org/licenses/by/4.0/), which permits unrestricted use, distribution, and reproduction in any medium, provided you give appropriate credit to the original author(s) and the source, provide a link to the Creative Commons license, and indicate if changes were made.

\section{References}

1. Hahn, S., Park, D.K., Bascunan, J., Iwasa, Y.: HTS Pancake coils without turn-to-turn insulation. IEEE Trans. Appl. Supercond. 21, 1592-1595 (2011)

2. Choi, S., Jo, H.C., Hwang, Y.J., Hahn, S., Ko, T.K.: A study on the no insulation winding method of the HTS coil. IEEE Trans. Appl. Supercond. 22 (2012)

3. Hahn, S., Kim, Y., Ling, J.Y., Voccio, J., Park, D.K., Bascunan, J., Shin, H.J., Lee, H., Iwasa, Y.: No-insulation coil under timevarying condition: magnetic coupling with external coil. IEEE Trans. Appl. Supercond. 23 (2013)

4. Wang, Y.W., Xu, D.Q., Sun, H., Liu, X., Sheng, J., Li, K., Hong, Z.Y., Jin, Z.J., Li, Z.Y.: Study on no-insulation hts pancake coils with iron core for superconducting DC induction heaters. IEEE Trans. Appl. Supercond. 25 (2015)

5. Wang, Y., Song, H., Xu, D., Li, Z.Y., Jin, Z., Hong, Z.: An equivalent circuit grid model for no-insulation HTS pancake coils. Supercond. Sci. Technol. 28 (2015)

6. Wang, X.D., Hahn, S., Kim, Y., Bascunan, J., Voccio, J., Lee, H., Iwasa, Y.: Turn-to-turn contact characteristics for an equivalent circuit model of no-insulation ReBCO pancake coil. Supercond. Sci. Technol. 26 (2013)

7. Wang, X., Wang, T., Nakada, E., Ishiyama, A., Itoh, R., Noguchi, S.: Charging behavior in no-insulation REBCO pancake coils. IEEE Trans. Appl. Supercond. 25 (2015)

8. Wang, T., Noguchi, S., Wang, X., Arakawa, I., Minami, K., Monma, K., Ishiyama, A., Hahn, S., Iwasa, Y.: Analyses of transient behaviors of no-insulation REBCO pancake coils during sudden discharging and overcurrent. IEEE Trans. Appl. Supercond. 25 (2015)

9. Zhang, M., Yuan, W.J., Kvitkovic, J., Pamidi, S.: Total AC loss study of $2 \mathrm{G}$ HTS coils for fully HTS machine applications. Supercond. Sci. Technol. 28 (2015)

10. Kim, K.L., Hahn, S., Kim, Y., Yang, D.G., Song, J.B., Bascunan, J., Lee, H., Iwasa, Y.: Effect of winding tension on electrical behaviors of a no-insulation ReBCO pancake coil. IEEE Trans. Appl. Supercond. 24 (2014)

11. Yoshitomi, E.S.O.K., Vyatkin, V.S., Kiuchi, M., Matsushita, T., Hamabe, M., Yamaguchi, S., Inada, R.: AC loss of ripple current in superconducting DC power transmission cable. In: Proceedings of the 26th International Symposium on Superconductivity, vol. 58 (2013)

12. Zhu, J.H., Zhang, Z.Y., Zhang, H.M., Zhang, M., Qiu, M., Yuan, W.J.: Electric measurement of the critical current, AC loss, and current distribution of a prototype HTS cable. IEEE Trans. Appl. Supercond. 24 (2014)

13. Norris, W.T.: Calculation of hysteresis losses in hard superconductors carrying $\mathrm{AC}$-isolated conductors and edges of thin sheets. J. Phys. D. Appl. Phys. 3 (1970)

14. Soldering Instructions for SuperPower2G HTS Wire available http://www.superpower-inc.com/system/files/SP_Soldering+ Instructions_2014_v1.pdf 